\title{
Prevalencia de sobrepeso, obesidad, actividad física y tabaquismo en adolescentes argentinos: Encuestas Mundiales de Salud Escolar y de Tabaco en Jóvenes, 2007-2012 \\ Prevalence of overweight, obesity, physical activity and tobacco use in Argentine youth: Global School-Based Student Health Survey and Global Youth Tobacco Survey, 2007-2012
}

Dr. Daniel Ferrante ${ }^{a}$, Dr. Bruno Linetzky ${ }^{a}$, Lic. Miguel Ponce ${ }^{a}$, Lic. Lucila Goldberg, Dr. Jonathan Konfino ${ }^{a}$ y Dr. Sebastián Laspiur ${ }^{a}$

\section{RESUMEN}

Introducción. En 2007 y 2012, se implementaron en nuestro país las Encuestas Mundiales deSalud en Jóvenes(EMSE) y de Tabaco en Jóvenes(EMTJ) para estimar la prevalencia de comportamientos de riesgo y factores de protección entre los adolescentes de 13 a 15 años.

Objetivo. Evaluar los cambios de los indicadores de alimentación y peso corporal, tabaco y actividad física en los últimos 5 años.

Metodología. Estudio de corte transversal. Se utilizó un muestreo aleatorizado bietápico con selección de 600 escuelas a nivel nacional. Los estudiantes de las divisiones elegidas al azar fueron invitados a responder el cuestionario autoadministrado (de la EMSE o de la EMTJ). Resultados. En 2012, la encuesta fue respondida por 20697 alumnos de 544 escuelas para la EMSE y 2062 para la EMTJ en 73 escuelas. Entre 2007 y 2012, se incrementó en forma significativa la prevalencia de sobrepeso $(24,5 \%$ en $2007,28,6 \%$ en 2012$)$ y obesidad $(4,4 \%$ en $2007,5,9 \%$ en 2012), y se mantuvieron elevados los consumos de bebidas azucaradas y comidas rápidas. Se observó una leve mejora en el nivel de actividad física (12,7\% en $2007,16,7 \%$ en 2012 ) aunque continúa por debajo de lo recomendado. Se redujo la prevalencia de tabaquismo $(24,5 \%$ en $2007,19,6 \%$ en 2012), pero persiste elevado el acceso a productos del tabaco y la exposición al humo de tabaco ajeno en lugares públicos, incluso la escuela.

Conclusiones. La progresión de la epidemia de sobrepeso y obesidad indica una necesidad de profundizar acciones en alimentación saludable y actividad física. A pesar del descenso de la prevalencia de tabaquismo, es necesario seguir fortaleciendo las acciones de control de tabaco.

Palabras clave: conducta adolescente, prevalencia, enfermedades no transmisibles, factores de riesgo, políticas públicas, encuestas de salud.

http:/ /dx.doi.org/10.5546/aap.2014.496

\section{INTRODUCCIÓN}

En la adolescencia, comienzan comportamientos que ponen en peligro el estado actual de salud y el futuro. Casi dos tercios de las muertes prematuras y un tercio de la carga total de morbilidad en adultos se asocian a condiciones o comportamientos que comenzaron en su juventud. ${ }^{1,2}$

La mayor parte de esta carga de enfermedad la manifiestan las enfermedades no transmisibles, que explican en Argentina el 63\% de las muertes. ${ }^{3}$ La mayor parte del riesgo atribuible de estas enfermedades está relacionado con la alimentación no saludable, el consumo de tabaco y la inactividad física. ${ }^{4}$

El sobrepeso y la obesidad están aumentando a nivel mundial, especialmente en países en desarrollo. Factores, como un mayor consumo de alimentos procesados y bebidas azucaradas y una reducción progresiva de la actividad física, contribuyen a este incremento. ${ }^{5}$

La gran mayoría de los fumadores comienza durante la adolescencia. Hoy en día, está aumentando a nivel mundial el número de adolescentes fumadores, especialmente entre las mujeres. ${ }^{6}$

La inactividad física se está incrementando en todo el mundo, debido al mayor acceso al automóvil, a condiciones laborales, la tecnología 
y otros determinantes. Durante la adolescencia, se adquieren las conductas que permiten llevar adelante una vida activa en el futuro, pero en forma progresiva los jóvenes están perdiendo incentivos. ${ }^{7,8}$

Para diseñar y monitorear acciones de promoción de la salud, es necesario contar con información válida y confiable. ${ }^{9}$ Para ello, se realizan encuestas periódicas en escuelas medias, habitualmente entre los 13 y los 15 años de edad, ya que es cuando comienzan muchas de las conductas de riesgo.

En este contexto, la Encuesta Mundial de Salud Escolar (EMSE) y la Encuesta Mundial de Tabaco en Jóvenes (EMTJ), impulsadas por los Ministerios de Salud y de Educación de la Nación, constituyen insumos básicos para la toma de decisiones.

En 2001, la Organización Mundial de la Salud (OMS), UNICEF, UNESCO, ONUSIDA y los Centros para el Control y Prevención de Enfermedades de Estados Unidos (CDC) desarrollaron la EMSE. Desde el año 2003, se ha utilizado en muchos países ${ }^{10}$ y se realizó en $2007^{11}$ y 2012 en Argentina.

La EMTJ forma parte del Sistema Global de Vigilancia de Tabaco y fue desarrollada por la OMS, el CDC y la Asociación de Salud Pública de Canadá. Se ha implementado en más de 170 países desde 1999. ${ }^{12}$

En nuestro país, se realizó la EMTJ en la Ciudad de Buenos Aires (2000, 2003 y 2007 y 2012) y la provincia de Buenos Aires (2003 y 2007 y 2012) y por primera vez a nivel nacional (200713 y 2012).

El objetivo de este informe es describir los resultados de ambas encuestas, que fueron realizadas en forma simultánea en noviembre de 2012 a nivel nacional, y comparar los factores de riesgo de enfermedades no transmisibles relevados por las mismas encuestas realizadas en 2007.14,15

\section{MÉTODOS}

Diseño: estudio descriptivo/analítico de tipo corte transversal.

\section{Población}

Alumnos de entre 13 y 15 años que cursan de $1^{\text {er }}$ a $3^{\text {er }}$ año de escuelas medias de todo el país.

\section{Instrumentos}

Para las encuestas a alumnos, se utilizaron los cuestionarios de la EMSE y la EMTJ. Ambas encuestas utilizan cuestionarios validados y cuentan con preguntas básicas comunes y preguntas específicas por país. Las preguntas fueron traducidas y adaptadas transculturalmente a nivel nacional. Las encuestas fueron autoadministradas. Cada alumno completó una grilla de respuestas anónima que se entregó al final de la hora de clase cuando se realizó la encuesta.

Aunque la EMSE y la EMTJ relevan múltiples aspectos, se incluyen en este artículo solamente alimentación, tabaco y actividad física.

Para el relevamiento del entorno escolar, el personal de la encuesta completó una ficha de observación directa y una encuesta breve a informantes clave de cada escuela.

\section{Aspectos incluidos y definición de variables}

Una descripción completa de las variables y su justificación puede encontrarse en múltiples publicaciones. ${ }^{10,12}$

\section{Alimentación y peso corporal:}

- Prevalencia de sobrepeso: definida como un índice de masa corporal mayor a 1 desvío estándar para cada grupo de edad y sexo, siguiendo las recomendaciones de la OMS ${ }^{16}$ por autorreporte de peso y altura.

- Prevalencia de obesidad: definida como un índice de masa corporal mayor a 2 desvíos estándar para cada grupo de edad y sexo, siguiendo las recomendaciones de la OMS por autorreporte de peso y altura.

- Consumo de frutas y verduras (5 o más veces diariamente en los últimos 30 días, incluye tubérculos).

- Consumo de gaseosas (2 o más veces diariamente en los últimos 30 días).

- Consumo de comidas rápidas (3 o más veces en todas las semanas en los últimos 30 días).

Consumo de tabaco:

- Prevalencia de tabaquismo: consumo de, al menos, un cigarrillo en los últimos 30 días.

- Prevalencia de exposición al humo de tabaco ajeno alguna vez en los últimos 7 días: en el hogar y fuera del hogar.

- Proporción de fumadores actuales que quieren dejar de fumar.

- Proporción de fumadores a quienes no se les negó la venta de cigarrillos por su edad en los últimos 30 días.

\section{Actividad física:}

- Proporción de jóvenes físicamente activos: más de 60 minutos diarios de actividad física en, al menos, 5 días de los últimos 7 días. 
- Proporción de jóvenes que permanecieron 3 o más horas sentados en un día típico (habitualmente).

- Proporción de jóvenes que recibieron, al menos, tres clases de actividad física por semana en el último año lectivo.

- Proporción de jóvenes que caminaron o fueron en bicicleta a la escuela 1 o más veces en los últimos 7 días.

\section{Diseño de la muestra}

Las encuestas EMSE y EMTJ de 2012 utilizaron un diseño de muestra bietápico para producir una muestra representativa de alumnos de $1^{\text {er }}$ a $3^{\text {er }}$ año de educación media a nivel nacional (de $8^{\circ}$ EGB a $1^{\circ}$ Polimodal en el caso de la provincia de Buenos Aires), la EMTJ a nivel nacional y la EMSE a nivel nacional y provincial.

La primera etapa del muestreo abarcó todas las escuelas, públicas o privadas, de educación media y utilizó el censo del Ministerio de Educación de la Nación. Las escuelas se seleccionaron con una probabilidad proporcional al número de alumnos. Se seleccionaron 600 escuelas para participar en la encuesta para la EMSE (ya que permite obtener estimaciones por provincia) y un total de 75 escuelas para la EMTJ.

Se realizó luego un muestreo sistemático en cada escuela para seleccionar las clases (divisiones o aulas), y se seleccionaron entre 2 y 3 clases por escuela. Todos los alumnos que asistieron en cada clase seleccionada el día del relevamiento eran aptos para participar en la encuesta.

Se aplicó un factor de ponderación para expandir la muestra de acuerdo con las probabilidades de selección de cada etapa y ajuste a la población general por sexo y edad y por no respuesta.

\section{Trabajo de campo y administración de las encuestas}

El trabajo de campo de la encuesta se llevó a cabo entre los meses de noviembre y diciembre de 2012. Los procedimientos de la encuesta fueron diseñados para proteger la intimidad de los alumnos, permitiendo una participación anónima y voluntaria. La encuesta fue autoadministrada.

\section{Evaluación del entorno escolar}

En una submuestra de 250 escuelas del país, se realizaron observaciones dentro de cada establecimiento, como aspectos relacionados con actividad física (cantidad de horas curriculares, oferta de cursos extracurriculares, instalaciones, etc.), tabaco (indicios de tabaco dentro de baños, aulas, pasillos, etc.) y oferta de alimentos en kioscos y/o comedores escolares.

\section{Análisis estadístico}

Se estimaron prevalencias para el total, ambos sexos y para 2007 y 2012 y sus intervalos de confianza del $95 \%$ teniendo en cuenta el diseño de muestra complejo utilizado. Se compararon las prevalencias entre 2007 y 2012 utilizando test de comparación de proporciones independientes de chi cuadrado, que incluyó el diseño de muestra complejo.

En la submuestra de escuelas, se reportó la proporción de escuelas que presentaba oferta de alimentos y bebidas, oportunidades e infraestructura para realizar actividad física, y cumplimiento de la prohibición de fumar en las escuelas.

Los valores de p iguales o menores a 0,05 se consideraron estadísticamente significativos.

\section{Consideraciones éticas}

El protocolo fue aprobado por un comité de ética independiente en Argentina, además de contar con la aprobación del Comité de Ética de la Organización Panamericana de la Salud. La realización de las encuestas contó con la autorización del Ministerio de Educación de la Nación, de los ministerios provinciales y de cada autoridad escolar.

Las respuestas fueron totalmente confidenciales y anonimizadas en hojas de respuesta sin identificación, que se recolectaban al finalizar la hora de clase. Se solicitó consentimiento oral a los alumnos antes de iniciar la encuesta autoadministrada.

\section{RESULTADOS}

Para EMSE, la tasa de respuesta fue $77 \%$ en 2007 (47 escuelas y 1498 alumnos) y 71\% en 2012 (544 escuelas y 20697 alumnos). En 2012, fue menor la respuesta a nivel escolar (91\% vs. $94 \%)$ y se redujo la respuesta a nivel individual $(73,4 \%$ vs. $79 \%$ ).

En la EMTJ, las tasas de respuesta fueron $74 \%$ en 2007 (71 escuelas y 3421 alumnos) y 76,9\% en 2012 (2062 alumnos en 73 escuelas).

Un total de 232 escuelas fueron incluidas en el relevamiento del entorno escolar. La distribución por edad y sexo de los encuestados de las muestras en 2007 y 2012 se describe en la Tabla 1 y la participación de escuelas y alumnos en 2012, en la Figura 1. 
TABla 1. Encuestas de Salud Escolar y Tabaco en Jóvenes

Encuesta Mundial de Salud Escolar, 2007 y 2012: muestra según edad y sexo

\begin{tabular}{llcc}
\hline Año & & $\mathbf{2 0 0 7}$ & $\mathbf{2 0 1 2}$ \\
\hline Grupos & & \multicolumn{3}{c}{ Frecuencia } \\
\hline Sexo & Masculino & 705 & 9671 \\
& Femenino & 793 & 11026 \\
Edad & 13 años & 333 & 4951 \\
& 14 años & 595 & 8091 \\
& 15 años & 570 & 7655 \\
\hline
\end{tabular}

Encuesta Mundial de Tabaco en Jóvenes, 2007 y 2012: muestra según edad y sexo

\begin{tabular}{llcc}
\hline Año & & $\mathbf{2 0 0 7}$ & $\mathbf{2 0 1 2}$ \\
\hline Grupos & & \multicolumn{3}{c}{ Frecuencia } \\
\hline Sexo & Masculino & 1612 & 1016 \\
& Femenino & 1809 & 1046 \\
Edad & 13 años & 765 & 594 \\
& 14 años & 1303 & 812 \\
& 15 años & 1353 & 656 \\
\hline
\end{tabular}

\section{Alimentación}

En 2012, 17,6\% del total consumió 5 porciones diarias de frutas y verduras, que se incrementó levemente desde 2007, cuando fue de $14 \%$ $(\mathrm{p}=0,041)$.

El 48,1\% del total consumió 2 o más veces por día bebidas azucaradas, con un leve descenso en relación con 2007( $\mathrm{p}=0,013)$.

No hubo cambios entre los dos años en el consumo de comidas rápidas (7,4\% en 2007 vs. $6,8 \%$ en 2012) (véase la Tabla 2).

En $80,2 \%$ de las escuelas, había un kiosco. El $81,2 \%$ ofrecía gaseosas no dietéticas, y solo $24,7 \%$ ofrecía frutas. El 91,4\% de los kioscos ofrecía alimentos, golosinas y bebidas con alta densidad calórica, sodio, azúcares y/o grasas (por ejemplo, snacks, hamburguesas y panchos, bebidas azucaradas, golosinas). El 90,9\% de las escuelas tenía agua potable en los baños, aunque solo 5,6\% tenía bebederos o dispensadores de agua.

Figura 1. Diagrama de flujo de participantes de la Encuesta Mundial de Salud en Jóvenes, 2012, y de la Encuesta Mundial de Tabaco en Jóvenes, 2012

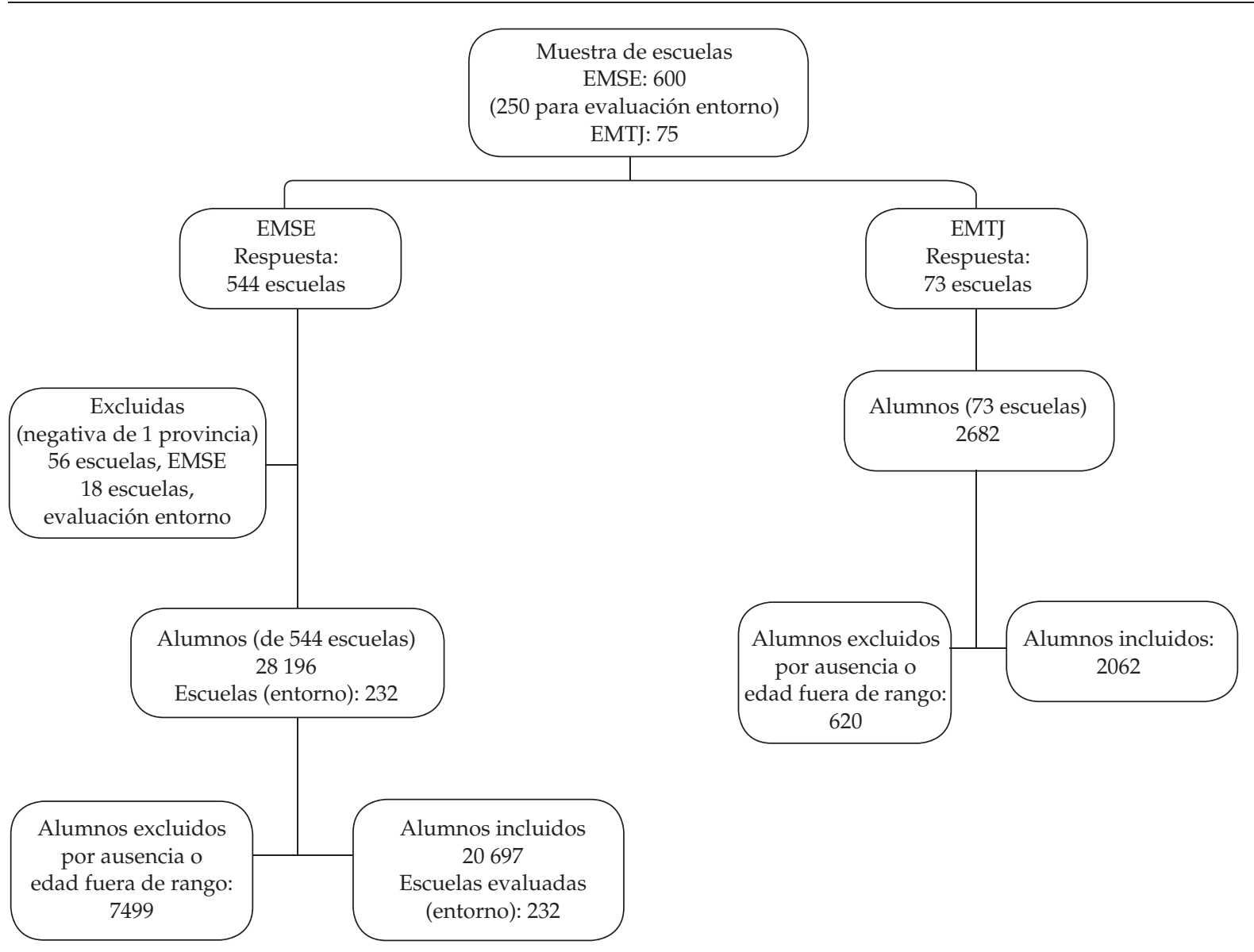




\section{Sobrepeso y obesidad}

La prevalencia de sobrepeso se incrementó de $24,5 \%$ en 2007 a $28,6 \%$ en 2012 ( $p=0,025)$, con mayor prevalencia en varones en ambos años. El 5,9\% presentó obesidad en 2012 (8,3\% en varones y 3,6\% en mujeres), que se incrementó significativamente $(p=0,05)$ en relación con 2007 , cuando fue de $4,4 \%$. Ambos sexos presentaron similares incrementos (Tabla 2).

\section{Actividad física}

Solo el $21,2 \%$ de los varones, $12,4 \%$ de las mujeres y $16,7 \%$ del total eran físicamente activos, algo mayor que en 2007, cuando $12,7 \%$ lo eran $(\mathrm{p}=0,014)$.

No hubo cambios en la proporción de jóvenes que pasaba 3 h o más sentados por día $(49,2 \%$ en 2007 vs. $50,3 \%$ en 2012) y en la proporción de jóvenes que se transportaba en forma activa a la escuela (74,3\% en 2007 vs. $68,8 \%$ en 2012).

Entre 2007 y 2012, se incrementó la proporción de jóvenes que reportó que en su escuela se impartían 3 o más clases semanales de actividad física, de $18,8 \%$ a $25,9 \%(\mathrm{p}=0,005)$ (Tabla 3$)$.

En relación con el relevamiento de escuelas, el $69 \%$ contaba con espacio propio para realizar actividad física. El $49 \%$ ofrecía actividades extracurriculares.

\section{Consumo de tabaco}

La prevalencia de tabaquismo (cigarrillos) descendió de forma significativa de $24,5 \%$ a $19,6 \%$ $(p=0,015)$, con mayor consumo en mujeres en ambos años $(p=0,002)$. Una mayor proporción de jóvenes fumadores deseó dejar de fumar ( $50,2 \%$ en 2007 vs. $55,2 \%$ en 2012). La exposición al humo de tabaco ajeno se redujo, a expensas de la exposición en el hogar ( $54,7 \%$ en 2007 vs. $47,5 \%$ en $2012, p=0,007$ ). A la mayoría de los jóvenes no se les niega la compra de cigarrillos, sin cambios significativos en el período evaluado $(88,7 \%$ en 2007 vs. $81,5 \%$ en 2012) (Tabla 4 ).

Por observación, en las escuelas, el 24,6\% presentó evidencias de consumo de cigarrillos. Solamente el 27,6\% presentó cartelería de prohibición de fumar.

\section{DISCUSIÓN}

Estas encuestas nacionales realizadas en 2012 muestran un incremento del sobrepeso y la obesidad, un consumo algo menor pero persistentemente elevado de bebidas azucaradas y comidas rápidas, un bajo consumo de frutas y verduras. En 2005, aunque en otra población, la Encuesta Nacional de Nutrición y Salud había reportado una prevalencia de obesidad en niños de $4,4 \%$ menores a 5 años y en mujeres de entre 10 y 49 años de $15,3 \%$.

El sobrepeso y la obesidad progresaron a pesar de las leves mejoras observadas en la alimentación, que continúa con un patrón lejano de lo recomendado.

Han contribuido en estos últimos años a generar un entorno obesogénico, probablemente

TABLA 2. Encuesta Mundial de Salud Escolar, alimentación y peso corporal, 2007-2012

\begin{tabular}{|c|c|c|c|c|c|c|c|}
\hline \multirow{3}{*}{$\begin{array}{l}\text { Alimentación } \\
\text { y peso corporal }\end{array}$} & \multicolumn{3}{|c|}{2007} & \multicolumn{3}{|c|}{2012} & \multirow{3}{*}{$\begin{array}{c}2007-2012 \\
P^{*}\end{array}$} \\
\hline & \multirow[t]{2}{*}{ Total } & \multicolumn{2}{|c|}{ Sexo } & \multirow[t]{2}{*}{ Total } & \multicolumn{2}{|c|}{ Sexo } & \\
\hline & & Varón & Mujer & & Varón & Mujer & \\
\hline $\begin{array}{l}\text { Consumo de frutas } \\
\text { y verduras } 5 \text { o } \\
\text { más veces al día } \\
\text { (últimos } 30 \text { días) }\end{array}$ & $\begin{array}{c}14,0 \% \\
(11,0 \%-17,7 \%)\end{array}$ & $\begin{array}{c}13,6 \% \\
(10,2 \%-18,0 \%)\end{array}$ & $\begin{array}{c}14,4 \% \\
(10,9 \%-18,7 \%)\end{array}$ & $\begin{array}{c}17,6 \% \\
(16,2 \%-19,1 \%)\end{array}$ & $\begin{array}{c}16,6 \% \\
(14,9 \%-18,4 \%)\end{array}$ & $\begin{array}{c}18,6 \% \\
(17,0 \%-20,4 \%)\end{array}$ & 0,041 \\
\hline $\begin{array}{l}\text { Consumo de } \\
\text { bebidas azucaradas } \\
2 \text { o más veces al día } \\
\text { (últimos } 30 \text { días) }\end{array}$ & $\begin{array}{c}52,6 \% \\
(48,9 \%-56,3 \%)\end{array}$ & $\begin{array}{c}50,5 \% \\
(46,2 \%-54,8 \%)\end{array}$ & $\begin{array}{c}54,5 \% \\
(49,0 \%-59,9 \%)\end{array}$ & $\begin{array}{c}48,1 \% \\
(46,0 \%-50,2 \%)\end{array}$ & $\begin{array}{c}54,0 \% \\
(50,5 \%-57,5 \%)\end{array}$ & $\begin{array}{c}49,8 \% \\
(47,5 \%-52,1 \%)\end{array}$ & 0,013 \\
\hline $\begin{array}{l}\text { Consumo en lugares } \\
\text { de comida rápida } \\
3 \text { o más días } \\
\text { (últimos } 7 \text { días) }\end{array}$ & $\begin{array}{l}7,4 \% \\
(4,7 \%-11,4 \%)\end{array}$ & $\begin{array}{c}7,0 \% \\
(4,2 \%-11,5 \%)\end{array}$ & $\begin{array}{c}7,3 \% \\
(4,5 \%-11,6 \%)\end{array}$ & $\begin{array}{c}6,8 \% \\
(5,8 \%-7,9 \%)\end{array}$ & $\begin{array}{c}7,0 \% \\
(5,4 \%-9,2 \%)\end{array}$ & $\begin{array}{c}6,6 \% \\
(5,8 \%-7,4 \%)\end{array}$ & 0,367 \\
\hline Sobrepeso & $\begin{array}{c}24,5 \% \\
(21,6 \%-28,1 \%)\end{array}$ & $\begin{array}{c}32,4 \% \\
(29,0 \%-36,0 \%)\end{array}$ & $\begin{array}{c}17,6 \% \\
(13,9 \%-23,0 \%)\end{array}$ & $\begin{array}{c}28,6 \% \\
(26,8 \%-30,4 \%)\end{array}$ & $\begin{array}{c}35,9 \% \\
(33,4 \%-38,4 \%)\end{array}$ & $\begin{array}{c}21,8 \% \\
(19,7 \%-24,1 \%)\end{array}$ & 0,025 \\
\hline Obesidad & $\begin{array}{c}4,4 \% \\
(3,2 \%-6,1 \%)\end{array}$ & $\begin{array}{c}6,5 \% \\
(4,5 \%-9,3 \%)\end{array}$ & $\begin{array}{c}2,6 \% \\
(1,3 \%-5,2 \%)\end{array}$ & $\begin{array}{c}5,9 \% \\
(5,1 \%-6,8 \%)\end{array}$ & $\begin{array}{c}8,3 \% \\
(7,1 \%-9,8 \%)\end{array}$ & $\begin{array}{c}3,6 \% \\
(2,8 \%-4,7 \%)\end{array}$ & 0,050 \\
\hline
\end{tabular}

* Corresponde a la comparación entre total del 2007 vs. total del 2012. 
por un mayor consumo de alimentos con alta densidad calórica y un bajo nivel de actividad física, que continúa bajo a pesar de haberse incrementado levemente. El consumo de bebidas azucaradas, aunque mostró una escasa reducción, sigue siendo una fuente importante de calorías en este grupo.

Se observó un incremento de adolescentes físicamente activos, aunque aún constituye un nivel bajo para lo recomendado a esta edad. Se observó un incremento de la oferta de clases de actividad física, pero esta herramienta no permite evaluar su intensidad ni calidad.

La prevalencia de tabaquismo se redujo en forma significativa, aunque persistió un mayor consumo en mujeres. Se incrementó la proporción de jóvenes que quieren dejar de fumar. Aunque ha mostrado una reducción significativa, continúa elevada la exposición al humo de tabaco ajeno. El acceso a productos de tabaco continúa siendo elevado. La prohibición de la publicidad y patrocinio de productos del tabaco (aunque

TABLA 3. Encuesta Mundial de Salud Escolar, actividad física, 2007-2012

\begin{tabular}{|c|c|c|c|c|c|c|c|}
\hline \multirow{3}{*}{$\begin{array}{l}\text { Actividad } \\
\text { física }\end{array}$} & \multicolumn{3}{|c|}{2007} & \multicolumn{3}{|c|}{2012} & \multirow{3}{*}{$\begin{array}{c}2007-2012 \\
P^{*}\end{array}$} \\
\hline & \multirow[t]{2}{*}{ Total } & \multicolumn{2}{|c|}{ Sexo } & \multirow[t]{2}{*}{ Total } & \multicolumn{2}{|c|}{ Sexo } & \\
\hline & & Varón & Mujer & & Varón & Mujer & \\
\hline $\begin{array}{l}\text { Físicamente activos, } \\
\text { al menos, } 60 \text { min por día } \\
\text { (últimos } 7 \text { días) }\end{array}$ & $\begin{array}{c}12,7 \% \\
(9,8 \%-16,3 \%)\end{array}$ & $\begin{array}{c}18,2 \% \\
(13,5 \%-24,2 \%)\end{array}$ & $\begin{array}{c}7,9 \% \\
(5,6 \%-11,2 \%)\end{array}$ & $\begin{array}{c}16,7 \% \\
(15,8 \%-17,7 \%)\end{array}$ & $\begin{array}{c}21,2 \% \\
(20,0 \%-22,6 \%)\end{array}$ & $\begin{array}{c}12,4 \% \\
(11,3 \%-13,7 \%)\end{array}$ & 0,014 \\
\hline $\begin{array}{l}\text { Pasó } 3 \text { o más horas } \\
\text { sentado (durante un } \\
\text { día típico usual) }\end{array}$ & $\begin{array}{c}49,2 \% \\
(41,6 \%-56,8 \%)\end{array}$ & $\begin{array}{c}44,0 \% \\
(37,1 \%-51,1 \%)\end{array}$ & $\begin{array}{c}53,9 \% \\
(44,4 \%-63,1 \%)\end{array}$ & $\begin{array}{c}50,3 \% \\
(48,6 \%-52,0 \%)\end{array}$ & $\begin{array}{c}47,1 \% \\
(44,3 \%-49,9 \%)\end{array}$ & $\begin{array}{c}53,4 \% \\
(51,1 \%-55,7 \%)\end{array}$ & 0,384 \\
\hline $\begin{array}{l}\text { Más de } 3 \text { clases } \\
\text { semanales }\end{array}$ & $\begin{array}{c}18,2 \% \\
(13,6 \%-22,7 \%)\end{array}$ & $\begin{array}{c}19,5 \% \\
(14,3 \%-24,7 \%)\end{array}$ & $\begin{array}{c}16,9 \% \\
(12 \%-21,9 \%)\end{array}$ & $\begin{array}{c}25,9 \% \\
(23,9 \%-28,0 \%)\end{array}$ & $\begin{array}{c}28,6 \% \\
(25,5 \%-32,0 \%)\end{array}$ & $\begin{array}{c}23,3 \% \\
(21,8 \%-24,9 \%)\end{array}$ & 0,005 \\
\hline $\begin{array}{l}\text { Transporte activo a } \\
\text { la escuela } 1 \text { o más } \\
\text { veces por semana } \\
\text { (últimos } 7 \text { días) }\end{array}$ & $\begin{array}{c}74,3 \% \\
(67,4 \%-80,2 \%)\end{array}$ & $\begin{array}{c}73,3 \% \\
(66,9 \%-78,8 \%)\end{array}$ & $\begin{array}{c}75,3 \% \\
(67,4 \%-81,8 \%)\end{array}$ & $\begin{array}{c}68,8 \% \\
(66,0 \%-71,5 \%)\end{array}$ & $\begin{array}{c}69,1 \% \\
(65,9 \%-72,2 \%)\end{array}$ & $\begin{array}{c}68,5 \% \\
(65,6 \%-71,3 \%)\end{array}$ & 0,255 \\
\hline
\end{tabular}

* Corresponde a la comparación entre total del 2007 vs. total del 2012.

Tabla 4. Encuesta Mundial de Tabaco en Jóvenes, consumo de tabaco, 2007-2012

\begin{tabular}{|c|c|c|c|c|c|c|c|}
\hline \multirow{3}{*}{$\begin{array}{l}\text { Consumo } \\
\text { de tabaco }\end{array}$} & \multicolumn{3}{|c|}{2007} & \multicolumn{3}{|c|}{2012} & \multirow{3}{*}{$\begin{array}{c}2007-2012 \\
P^{*}\end{array}$} \\
\hline & \multirow[t]{2}{*}{ Total } & \multicolumn{2}{|c|}{ Sexo } & \multirow[t]{2}{*}{ Total } & \multicolumn{2}{|c|}{ Sexo } & \\
\hline & & Varón & Mujer & & Varón & Mujer & \\
\hline $\begin{array}{l}\text { Porcentaje de jóvenes } \\
\text { que actualmente fuma } \\
\text { cigarrillos }\end{array}$ & $\begin{array}{c}24,5 \% \\
(22,2 \%-27 \%)\end{array}$ & $\begin{array}{c}21,1 \% \\
(18,5 \%-23,8 \%)\end{array}$ & $\begin{array}{c}27,3 \% \\
(23,4 \%-31,6 \%)\end{array}$ & $\begin{array}{c}19,6 \% \\
(16,4 \%-23,3 \%)\end{array}$ & $\begin{array}{c}17,4 \% \\
(14,7 \%-20,5 \%)\end{array}$ & $\begin{array}{c}21,5 \% \\
(17,1 \%-26,7 \%)\end{array}$ & 0,015 \\
\hline $\begin{array}{l}\text { Deseo de dejar } \\
\text { de fumar ahora }\end{array}$ & $\begin{array}{c}50,2 \% \\
(44,1 \%-56,2 \%)\end{array}$ & $\begin{array}{c}47,3 \% \\
(38 \%-56,9 \%)\end{array}$ & $\begin{array}{c}52,3 \% \\
(45,7 \%-58,9 \%)\end{array}$ & $\begin{array}{c}55,2 \% \\
(44,7 \%-65,3 \%)\end{array}$ & $\begin{array}{c}57,8 \% \\
(44,9 \%-69,7 \%)\end{array}$ & $\begin{array}{c}53,1 \% \\
(38,4 \%-67,3 \%)\end{array}$ & 0,420 \\
\hline $\begin{array}{l}\text { Fumadores actuales } \\
\text { de cigarrillos a los que no } \\
\text { se impidió la compra de } \\
\text { cigarrillos en los últimos } \\
30 \text { días debido a su edad }\end{array}$ & $\begin{array}{c}88,7 \% \\
(83,1 \%-92,6 \%)\end{array}$ & $\begin{array}{c}86,4 \% \\
(75,4 \%-93,0 \%)\end{array}$ & $\begin{array}{c}90,9 \% \\
(84,0 \%-95,0 \%)\end{array}$ & $\begin{array}{c}81,5 \% \\
(72,7 \%-88,0 \%)\end{array}$ & $\begin{array}{c}79,3 \% \\
(68,1 \%-87,3 \%)\end{array}$ & $\begin{array}{c}82,9 \% \\
(72,7 \%-89,8 \%)\end{array}$ & 0,176 \\
\hline Exposición en el hogar & $\begin{array}{c}54,7 \% \\
(51,9 \%-57,4 \%)\end{array}$ & $\begin{array}{c}51,2 \% \\
(48,3 \%-55,2 \%)\end{array}$ & $\begin{array}{c}57,7 \% \\
(54,4 \%-61,0 \%)\end{array}$ & $\begin{array}{c}47,5 \% \\
(43,1 \%-51,9 \%)\end{array}$ & $\begin{array}{c}44,5 \% \\
(39,7 \%-49,4 \%)\end{array}$ & $\begin{array}{c}50,1 \% \\
(44,6 \%-55,7 \%)\end{array}$ & 0,007 \\
\hline Fuera del hogar & $\begin{array}{c}68,6 \% \\
(65,9 \%-71,1 \%)\end{array}$ & $\begin{array}{c}66,4 \% \\
(63,2 \%-69,5 \%)\end{array}$ & $\begin{array}{c}70,7 \% \\
(66,9 \%-74,2 \%)\end{array}$ & $\begin{array}{c}69,4 \% \\
(65,7 \%-72,9 \%)\end{array}$ & $\begin{array}{c}64,2 \% \\
(60,0 \%-68,2 \%)\end{array}$ & $\begin{array}{c}74,3 \% \\
(69,4 \%-78,6 \%)\end{array}$ & 0,732 \\
\hline
\end{tabular}

* Corresponde a la comparación entre total del 2007 vs. total del 2012. 
persiste la publicidad en sitios de venta), las normativas libres de humo tanto provinciales como nacional y la aparición de advertencias sanitarias gráficas pueden explicar esta reducción. Sin embargo, la persistencia de precios bajos de cigarrillos genera una accesibilidad elevada en jóvenes, considerando también que no resultan efectivas las prohibiciones de venta a menores. Además de profundizar las acciones actuales, es necesario lograr un incremento de los precios de cigarrillos, que es especialmente efectivo para reducir el consumo en esta población. ${ }^{17}$ Argentina cuenta en este momento con uno de los cigarrillos más económicos de la región ajustado al poder de compra nacional. ${ }^{18}$

En relación con el sobrepeso y la obesidad, se lanzó recientemente la Guía Nacional de Obesidad. ${ }^{19}$ Para la promoción de una alimentación saludable en especial a este grupo poblacional y en el entorno escolar, han avanzado en nuestro país iniciativas de kioscos y cantinas saludables. A nivel poblacional, cambios en la demanda (como estrategias de educación alimentaria, ${ }^{20,21}$ regulación de la comercialización de alimentos, ${ }^{22}$ cambios en el etiquetado nutricional) ${ }^{23} \mathrm{y}$ en la oferta (regulación del contenido de nutrientes de alimentos, como sodio, ${ }^{24}$ grasas trans ${ }^{25}$ azúcares, políticas impositivas, ${ }^{26,27}$ tamaños de porciones) deberían profundizarse para promover una alimentación más saludable.

En relación con la actividad física, existe evidencia consistente sobre incrementar las horas e intensidad de la actividad física realizada en las escuelas, ${ }^{28}$ promover actividades extracurriculares en las escuelas, acciones de comunicación, ${ }^{29}$ mayor acceso a oferta social de actividades físicas, ${ }^{30}$ mayor acceso a lugares públicos aptos para la actividad física y modificaciones del entorno urbano y promoción del transporte público y activo. ${ }^{31}$

Las limitaciones están relacionadas con la evaluación por autorreporte vs. medición directa del peso corporal y altura, y una tasa de respuesta menor a la esperada para EMSE debido a la negativa de una jurisdicción para realizar las encuestas y al ausentismo en algunas escuelas. La fortaleza de estas encuestas se relaciona con la utilización de cuestionarios validados, muestreo probabilístico y sustentabilidad en la metodología que permite caracterizar la evolución temporal.

Argentina no escapa a la epidemia de sobrepeso y obesidad y al sedentarismo que se está desarrollando en especial en países en vías de desarrollo. Profundizar las acciones de promoción de la salud tanto a nivel comunitario como escolar es necesario para comenzar a torcer esta tendencia. A pesar de las mejoras en el consumo de tabaco, es necesario fortalecer las políticas existentes en relación con precios, ambientes libres de humo y publicidad.

\section{CONCLUSIÓN}

Los principales resultados de estas encuestas indican que se ha reducido el consumo de tabaco, aunque sigue siendo elevada la exposición al humo de tabaco ajeno; la prevalencia de sobrepeso y obesidad están en aumento; y no se han observado mejoras muy significativas en alimentación saludable y actividad física.

\section{REFERENCIAS}

1. World Health Organization. Adolescents: health risks and solutions. Fact sheet $N^{\circ} 345$. Geneva:WHO;2014. Disponible en:http://www.who.int/mediacentre/factsheets/fs345/ en/index.html. [Consulta: 3 de marzo de 2014].

2. Gore FM, Bloem DJ, Patton GC, Ferguson J, et al. Global burden of disease in young people aged 10-24 years: a systematic analysis. Lancet 2011;377(9783):2093-102.

3. Secretaría de Políticas, Regulación e Institutos. Dirección de Estadísticas e Información deSalud. Estadísticas vitales. Información básica año 2012. Buenos Aires: Ministerio de Salud de la Nación; 2013. Disponible en: http://www. deis.gov.ar/Publicaciones / Archivos/Serie5Nro56.pdf. [Consulta: 3 de marzo de 2014].

4. Lim SS, Vos T, Flaxman AD, Danaei G, et al. A comparative risk assessment of burden of disease and injury attributable to 67 risk factors and risk factor clusters in 21 regions, 19902010: a systematic analysis for the Global Burden of Disease Study 2010. Lancet 2012;380(9859):2224-60.

5. McAllister EJ, Dhurandhar NV, Keith SW, Aronne LJ, et al. Ten putative contributors to the obesity epidemic. Crit Rev Food Sci Nutr 2009;49(10):868-913.

6. Warren CW, Jones NR, Eriksen MP, Asma S, et al. Patterns of global tobacco use in young people and implications for future chronic disease burden in adults. Lancet 2006;367(9512):749-53.

7. Bauman AE, Reis RS, Sallis JF, Wells JC, et al. Correlates of physical activity: why are some people physically active and others not? Lancet 2012;380(9838):258-71.

8. Sixty-fourth World Health Assembly. Youth and health risks. WHA 64.28. Geneva: World Health Organization; 2011. Disponible en: http://apps.who.int/gb/ebwha/ pdf_files/WHA64/A64_R28-en.pdf.[Consulta:3 demarzo de 2014].

9. Argentina. Ministerio de Salud de la Nación. Estrategia Nacional para la Prevención y Control de Enfermedades No Transmisibles. Buenos Aires: Ministerio de Salud de la Nación; 2009. Disponible en: http://www.msal.gov. ar/images/stories/bes/graficos/0000000203cnt-2013-07_ estrategia-nacional-prevencion-control-ent-as.pdf. [Consulta: 3 de marzo de 2014].

10. World Health Organization. Global school-based student health survey (GSHS) purpose and methodoloy. Geneva: WHO; 2014. Disponible en: http://www.who.int/chp/ gshs/methodology/en/. [Consulta: 3 de marzo de 2014].

11. Linetzky B, Morello P, Virgolini M, Ferrante D. Resultados de la Primera Encuesta Nacional de Salud Escolar. Argentina, 2007. Arch Argent Pediatr 2011;109(2):111-6. 
12. Centers of Disease Control and Prevention. Global Youth Tobacco Survey (GYTS)-Overview. Atlanta: CDC; 2014. Disponible en: http://nccd.cdc.gov/gtssdata/Ancillary/ Documentation.aspx?SUID=1\&DOCT=1. [Consulta: 3 de marzo de 2014].

13. Linetzky B, Mejia R, Ferrante D, De Maio FG, et al. Socioeconomic status and tobacco consumption among adolescents: a multilevel analysis of Argentina's Global Youth Tobacco Survey. Nicotine Tob Res 2012;14(9):1092-9.

14. Argentina. Ministerio de Salud de la Nación. Encuesta Mundial de Salud Escolar. Resultados de 2007. Argentina. Buenos Aires, 2008. [Consulta: 4 de marzo de 2014]. Disponible en: http://www.msal.gov.ar/ent/images/ stories/vigilancia/pdf/encuesta_mundial_salud_ escolar_2007.pdf.

15. Argentina. Ministerio de Salud de la Nación. Encuesta Mundial de Tabaquismo en Adolescentes en Argentina. Resultados de 2007 y comparación con encuestas previas. Argentina. Buenos Aires, 2009. [Consulta: 4 de marzo de 2014]. Disponible en: http://www.msal.gov.ar/ent/ images/stories/vigilancia/pdf/encuesta_tabaquismo_ adolescentes_2007.pdf.

16. World Health Organization. Grouth reference 5-19 years. BMI-for-age (5-19 years). Geneva: WHO; 2007. Disponible en: http:/ / www.who.int/growthref/who2007_bmi_for_ age/en/. [Consulta: 3 de marzo de 2014].

17. Martínez E, Mejia R, Pérez-Stable EJ. An empirical analysis of cigarette demand in Argentina. Tob Control 2013. Publicación electrónica 12 de junio de 2013. [En prensa].

18. Konfino J, Ondarsuhu D, Goldberg L, Linetzky B, et al. Encuesta mundial de tabaquismo en adultos 2012: resultados de la primera implementación en Argentina. Rev Argent Salud Publ 2013;4(16):6-15.

19. Argentina. Ministerio de Salud de la Nación. Guía de Práctica Clínica Nacional sobre Diagnóstico y Tratamiento de la Obesidad. Buenos Aires: Ministerio de Salud de la Nación; 2013. Disponible en: http://www.msal.gov.ar/ ent/images/stories/equipos-salud/pdf/2014-05_gpc_ obesidad.pdf. [Consulta: 3 de marzo de 2014].

20. Thorogood M, Simera I, Dowler E, Summerbell C, et al. A systematic review of population and community dietary interventions to prevent cancer. Nutr Res Rev 2007;20(1):74-88.

21. Wakefield MA, Loken B, Hornik RC. Use of mass media campaigns to change health behaviour. Lancet 2010;376(9748):1261-71.
22. Organización Mundial de la Salud. Conjunto de recomendaciones sobre la promoción de alimentos y bebidas no alcohólicas dirigida a los niños. Ginebra: OMS; 2010. Disponible en: http://whqlibdoc.who. int/publications / 2010/9789243500218_spa.pdf?ua $=1$. [Consulta: 3 de marzo de 2014].

23. Campos S, Doxey J, Hammond D. Nutrition labels on prepackaged foods: a systematic review. Public Health Nutr 2011;14(8):1496-506.

24. Ferrante D, Konfino J, Mejía R, Coxson P, et al. Relación costo-utilidad de la disminución del consumo de sal y su efecto en la incidencia de enfermedades cardiovasculares en Argentina. Rev Panam Salud Publica 2012;32(4):274-80.

25. Argentina. Ministerio de Salud de la Nación. Campaña "Argentina 2014 Libre de Grasas Trans". Buenos Aires: Ministerio de Salud de la Nación; 2014. Disponible en: http:/ / www.msal.gov.ar/ent/index.php?option=com_co ntent\&view=article\&id=348:campana-qargentina-2014libre-de-grasas-trans\&catid=9. [Consulta: 3 de marzo de 2014].

26. Maniadakis N, Kapaki V, Damianidi L, Kourlaba G. A systematic review of the effectiveness of taxes on nonalcoholic beverages and high-in-fat foods as a means to prevent obesity trends. Clinicoecon Outcomes Res 2013;5:51943.

27. Powell LM, Chriqui JF, Khan T, Wada R, et al. Assessing the potential effectiveness of food and beverage taxes and subsidies for improving public health: a systematic review of prices, demand and body weight outcomes. Obes Rev 2013;14(2):110-28.

28. Lonsdale C, Rosenkranz RR, Peralta LR, Bennie A, et al. A systematic review and meta-analysis of interventions designed to increase moderate-to-vigorous physical activity in school physical education lessons. Prev Med 2013;56(2):152-61.

29. Kahn EB, Ramsey LT, Brownson R, Heth GW, et al. The effectiveness of interventions to increase physical activity: a systematic review. Am J Prev Med 2002;22(4 Suppl):73-107.

30. Increasing physical activity. A report on recommendations of the Task Force on Community Preventive Services. MMWR Recomm Rep 2001;50(RR-18):1-14.

31. Heath GW, Brownson RC, Kruger J, Miles R, et al. The effectiveness of urban design and land use and transport policies and practices to increase physical activity: a systematic review. J Phys Act Health 2006;3 (Suppl1):S55-S76. 